\title{
Recent progress in flexible tactile sensor systems: from design to application
}

\author{
Jiefei Zhu', Changjian Zhou'², Min Zhang ${ }^{1}$ \\ ${ }^{1}$ School of Electronic and Computer Engineering, Peking University, Shenzhen 518055, China. \\ ${ }^{2}$ School of Microelectronics, South China University of Technology, Guangzhou 510641, China.
}

Correspondence to: Dr. Min Zhang, School of Electronic and Computer Engineering, Peking University, Shenzhen 518055, China. E-mail: zhangm@ece.pku.edu.cn

How to cite this article: Zhu J, Zhou C, Zhang M. Recent progress in flexible tactile sensor systems: from design to application. Soft Sci 2021;1:3. https://dx.doi.org/10.20517/ss.2021.02

Received: 23 May 2021 First Decision: 17 Jun 2021 Revised: 30 Jun 2021 Accepted: 9 Jul 2021 First online: 9 Jul 2021

Academic Editor: Zhifeng Ren Copy Editor: Xi-Jun Chen Production Editor: Xi-Jun Chen

\begin{abstract}
With the rapid development of artificial intelligence, human-machine interaction, and healthcare systems, flexible tactile sensors have huge market potentials and research needs, so that both fundamental research and application demonstrations are evolving rapidly to push the potential to reality. In this review, we briefly summarize the recent progress of the flexible tactile sensor system, including the common sensing mechanisms, the important performance evaluation parameters, the device design trend, and the main applications. Moreover, the current device design trend towards flexible tactile sensor systems is discussed, including novel structures for outstanding performance, sensor arrays for large-area information acquisition, multi-mode information acquisition, and integration of tactile sensors with transistors. Various emerging applications enabled with these sensors are also exemplified in this review to show the potentials of the tactile sensors. Finally, we also discuss the technical demands and the future perspectives of flexible tactile sensor systems.
\end{abstract}

Keywords: Tactile sensor, flexible sensing, sensor system

\section{INTRODUCTION}

Tactile sensation is the basic perception ability of human skin, which is a kind of feeling produced by tactile receptors in response to external stimuli. There is a tactile cone in the deep layer of the skin, where the various tactile mechanoreceptors respond to the pressure exerted on the skin surface and generate a small 
current signal, which is immediately transported to the brain through the nerve fibers. The sense of touch is enabled by distinguishing the magnitude and position of the signal ${ }^{[1]}$. The touch of the finger is the most sensitive as a result of the maximum contact density distributed on the fingertip, providing one of the most direct resourceful means for the human to obtain interactive information from the environment. Humans can feel the temperature, humidity, material type, shape, surface texture, hardness, vibration, and other information of the target object through touch. However, the tactile sensation of human skin cannot quantitatively gain the specific sensing parameters. Mimicking the functionality of human skin, a tactile sensor is a sensing system that can detect pressure, strain, temperature, humidity, and other information about an object. The measured information is converted into electrical signals and analyzed by an external processing circuit to obtain a more accurate tactile detection capability than human $\operatorname{skin}^{[2]}$. The tactile sensor needs to be flexible to adapt to objects with different surface morphologies and shapes, which is an important difference between the tactile sensor and the traditional sensor.

In recent years, with the rapid development of flexible electronics and the rapid popularization of intelligent terminals, the flexible tactile sensing technology has been developed rapidly and widely used in intelligent robots, human-machine interfaces, virtual and augmented reality, medical treatment, health monitoring, and other emerging applications [Figure 1]. Meanwhile, to satisfy the requirements of numerous emerging applications, flexibility, sensitivity, sensing range, response time, stability, and other performance parameters of flexible tactile sensors are also continuously improved ${ }^{[3-19]}$. Through the optimization of sensing material and device design, an ultra-thin sensor was realized without any sensory interference ${ }^{[20]}$. A multi-mode integrated tactile sensor system that can detect strain, temperature, and gas simultaneously was reported $^{[21]}$. Wireless tactile sensors were enabled with the development of Bluetooth transmission and near field communication technology ${ }^{[22-24]}$. To extend the application fields, a sensor array was realized to decode the facial strains through machine learning ${ }^{[25]}$, and a sweat sensor that can detect blood glucose, nicotine, and other health information was developed ${ }^{[26-28]}$. Thus, flexible tactile sensing has sufficient momentum of development and huge market potentials. Therefore, both fundamental research and application demonstration are evolving rapidly to push the potential to reality.

In this review, we summarize the recent progress of the flexible tactile sensor systems. After a short introduction about the common sensing mechanisms, the important parameters to evaluate the sensor performance are summarized, with typical data reported for different mechanisms listed for comparison. The development trend of tactile sensor systems is covered in Section 3. Starting from the performance optimization for a single tactile sensor emphasizing certain performance indicators, we further discuss the multi-mode tactile sensor aiming to obtain multiple sensing capabilities and the integration of processing circuits with the tactile sensor systems. The outstanding properties of the tactile sensors cover ultra-high sensitivity, self-powered operation, wireless connection, self-repairing capability, degradable material system, good biocompatibility, and nontoxicity. These properties enable wide applications including virtual and augmented reality, intelligent robots, human-machine interfaces, medical treatments, and wearable health monitoring, which are discussed in Section 4. Finally, we address the potential development trends and remaining challenges for the flexible tactile sensor systems in future research.

\section{WORKING PRINCIPLES OF THE FLEXIBLE TACTILE SENSORS}

\section{Sensing mechanisms}

Tactile sensors usually convert tactile information into electrical signals. They can be classified as piezoresistive, piezoelectric, capacitive, inductive, electromagnetic, photoelectric, and triboelectric types depending on the transducing mechanisms. The most widely used four types of tactile sensors are piezoresistive, capacitive, piezoelectric, and triboelectric, which are briefly reviewed as follows. The basic 


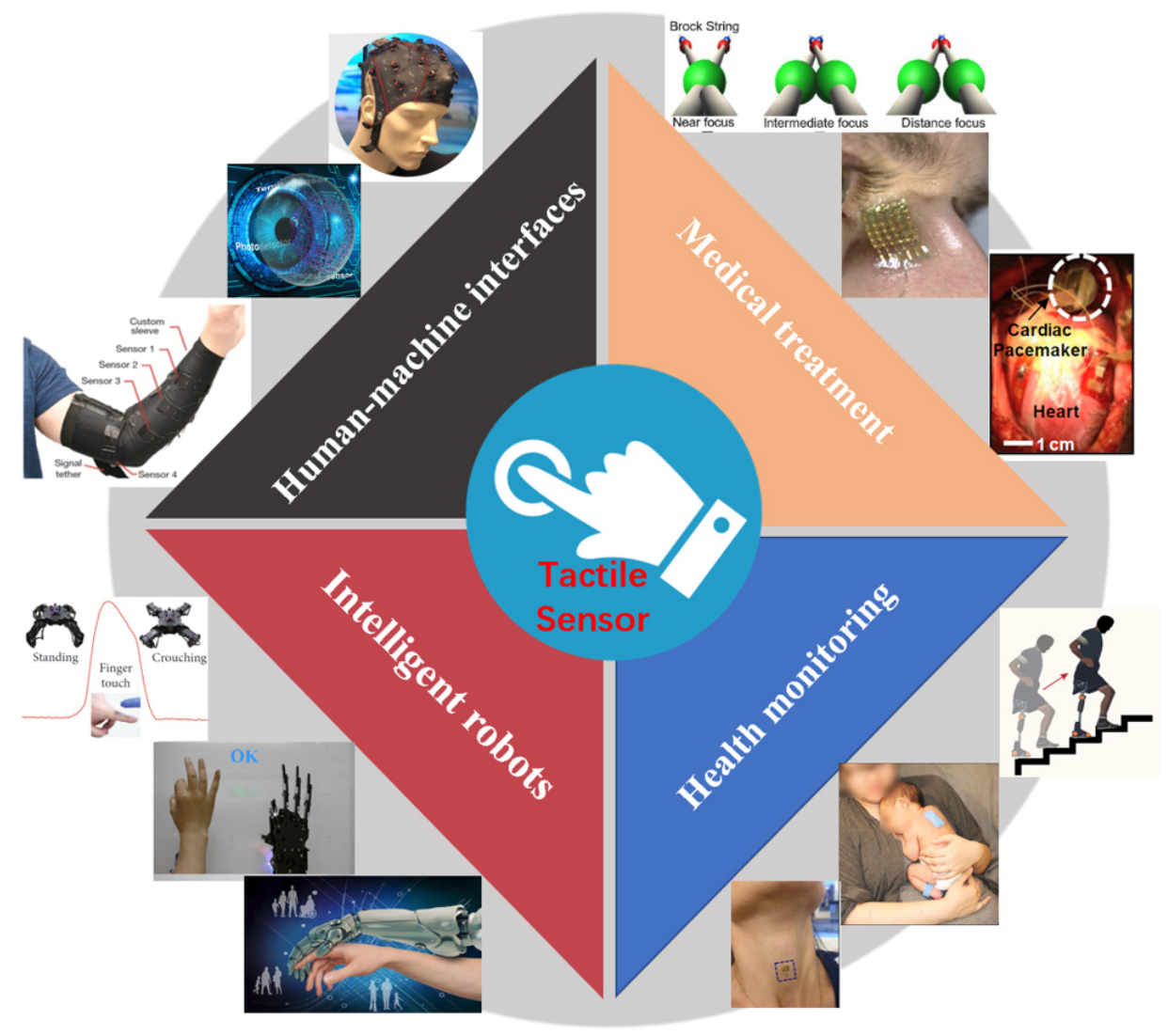

Figure 1. Diagram of the application of flexible tactile sensors. Human-machine interfaces. The brain-computer interfaces. Reproduced with permission from Ref. ${ }^{[97]}$. Copyright ${ }^{\complement}$ 2018. AAAS. Integrated contact lens sensor system. Reproduced with permission from Ref. ${ }^{[94]}$. Copyright ${ }^{\odot}$ 2021. Elsevier. Constructed sensor sleeve. Reproduced with permission from Ref. ${ }^{[105]}$. Copyright ${ }^{\odot}$ 2020. Springer Nature; Intelligent robots. Microrobots, reproduced with permission from Ref. ${ }^{[96]}$. Copyright ${ }^{\odot} 2020$. OAE Publishing Inc. Robot hand responding to "ok" gesture. Reproduced with permission from Ref. ${ }^{[57]}$. Copyright ${ }^{\odot} 2021$. Elsevier. Social robotics. Reproduced with permission from Ref. ${ }^{[97]}$. Copyright ${ }^{\oplus}$ 2018. AAAS; Health monitoring. Tactile sensor for speaking pressure monitoring. Reproduced with permission from Ref. ${ }^{[52]}$. Copyright ${ }^{\complement}$ 2014. Springer Nature. A wireless sensor system for health monitoring in neonatal. Reproduced with permission from Ref. ${ }^{[23]}$. Copyright ${ }^{\odot}$ 2021. Springer Nature. Flexible exoskeleton. Reproduced with permission from Ref. ${ }^{[100]}$. Copyright ${ }^{\odot}$ 2021. AAAS; Medical treatment. A piezoelectric sensor for energy harvesting in the operation of heart pacemakers. Reproduced with permission from Ref. ${ }^{[54]}$. Copyright ${ }^{\odot}$ 2014. PNAS. Eye astigmatism treatment system. Reproduced with permission from Ref. ${ }^{[103]}$. Copyright ${ }^{\odot}$ 2020. AAAS.

mechanisms of these four types are briefly shown in Figure 2.

\section{Piezoresistive mechanism}

The piezoresistive tactile sensor is built based on the strain-induced resistance variation, which can be easily detected by the resistance measurement system, as shown in Figure 2A. Generally, the resistance (R) of the piezoresistive tactile sensor is defined as $\mathrm{R}=\rho \mathrm{L} / \mathrm{A}$, where $\rho$ is the resistivity. $\mathrm{L}$ and A denote the length and area of the resistor, respectively. In response to the load applied by the contact operation, the change of the sensor resistance is of the following form:

$d R=(\rho / A) \times d L-\left[(\rho L) / A^{2}\right] \times d A+(L / A) \times d \rho$

The first two terms describe the resistance change due to the differential geometrical deformation effect, and the last term describes the resistance change due to the piezoresistive effect. For metallic conductors, the geometrical deformation effect usually dominates, while some semiconducting materials could exhibit a 
A

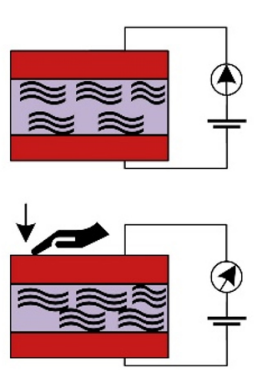

B

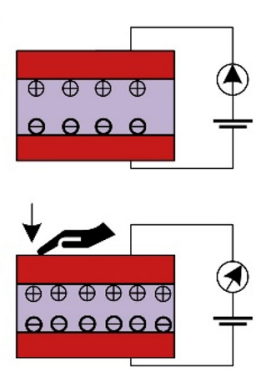

C
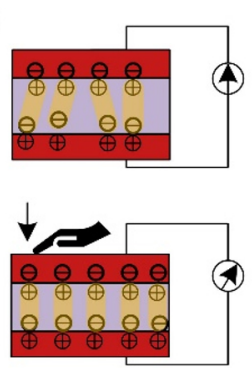

D

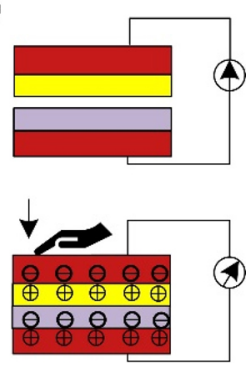

Figure 2. Schematic illustrations of four sensing mechanisms: (A) piezoresistive; (B) capacitive; (C) piezoelectric; and (D) triboelectric.

large piezoresistive effect, with which the order of magnitude for the signal is larger than that induced by the pure geometrical deformation. While the above equation can be used to explain the resistance change of a resistor formed by a single type of material, other factors also play an important effect for resistive tactile sensors, as the composition of the resistor includes both polymeric and inorganic nanomaterials. The change of the contact resistance between two conductive materials, and the change of conductive paths in conductive composites vary with the deformation of the flexible structures. For example, Xie et al. ${ }^{[29]}$ embedded vertical aligned multi-walled carbon nanotube (CNT) arrays into the polydimethylsiloxane (PDMS) and bonded two CNT/PDMS films face to face to realize a tunable range piezoresistive tactile sensor by adjusting the thickness of the soft spacer between the two CNT/PDMS films. The resistance variation mainly results from increasing CNT contact area with applied pressure ${ }^{[29]}$.

The piezoresistive tactile sensor has been widely studied and applied due to its simple structure design and readout mechanism. The piezoresistive tactile sensors have the advantages of simple structure, easy fabrication, low cost, high sensitivity ${ }^{[30,31]}$, and large measurement range, while there are also several drawbacks, such as obvious hysteresis effect, poor long-term stability, and requirement for external power supply, which are worthy of further investigating. The piezoresistive materials commonly used in piezoresistive tactile sensors include monocrystalline silicon $^{[32]}$, carbon nanotubes ${ }^{[29,33-35]}$, carbon black $^{[36]}$, graphene ${ }^{[37,38]}, \operatorname{MoS}_{2}^{[39]}$, and conductive polymers ${ }^{[00,41]}$.

\section{Capacitive mechanism}

The capacitive tactile sensor is based on the principle of capacitance change when the sensing structure responds to a mechanical stimulus, as shown in Figure $2 \mathrm{~B}$. The capacitance $(\mathrm{C})$ of the device can be calculated by using the parallel-plate capacitor model: $\mathrm{C}=\varepsilon_{0} \varepsilon_{\mathrm{r}} \mathrm{A} / \mathrm{s}$, where $\varepsilon_{0}$ is the vacuum dielectric constant, $\varepsilon_{\mathrm{r}}$ is the relative dielectric constant of the material, $\mathrm{A}$ is the overlap area of upper and lower parallel plates, and $s$ is the spacing between the upper and lower electrodes. Typical structures adopted in tactile sensors include the planar interdigital electrode/dielectric material and electrode/dielectric material/electrode sandwich structures. The differential capacitance of the sensing capacitor is given by:

$d C=\left[\left(\varepsilon_{0} \varepsilon_{r}\right) / s\right] \times d A-\left[\left(\varepsilon_{0} \varepsilon_{r} A\right) / s^{2}\right] \times d s+\left[\left(\varepsilon_{0} A\right) / s\right] \times d \varepsilon_{r}$

Similarly, the first two terms describe the contribution by the geometrical deformation, while the last term describes the contribution by the material dielectric constant. As flexible dielectric materials usually exhibit a small Young's modulus, a large deformation can be induced by a gentle touch operation, resulting in a large differential capacitance. 
Compared with the piezoresistive sensor, the sensitivity of the traditional capacitance sensor is relatively low, but it can be enhanced by using different micro/nanostructures. As-fabricated, the nanostructured capacitor features an air gap between pillars sandwiched between the upper and lower electrodes, so that both the overlapped area and distance, as well as the effective dielectric constant, are changed with applied pressure $^{[42]}$. Beyond the traditional capacitive sensor, the ionic sensor based on ion-interfacial sensing has been widely investigated in recent years because of its unique double electrode layer structure ${ }^{[43-45]}$. For instance, Bai et al.$^{[4]]}$ reported an ionic sensor based on a graded intrafillable architecture with a high sensitivity $\left(>220 \mathrm{kPa}^{-1}\right)$ in an ultra-broad sensing range (0.08 $\left.\mathrm{Pa}-360 \mathrm{kPa}\right)$. The advantages of capacitive tactile sensors include low energy consumption, independence of the operating temperature, and good stability. However, its initial capacitance is very small so that it is very easy to be interfered with by the parasitic capacitance of the testing circuit, resulting in a poor signal-to-noise ratio. The dielectric materials commonly used in capacitance tactile sensors include air gap ${ }^{[42]}$, ionic conductor ${ }^{[43-45]}, \mathrm{TiO}_{2}^{[46]}$, polyimide ${ }^{[47]}$, elastomers ${ }^{[4,49]}$, and $3 \mathrm{D}$ fabrics ${ }^{[50,51]}$.

\section{Piezoelectric mechanism}

The piezoelectric tactile sensor is based on the piezoelectric effect, that is, generating electric displacement in response to the applied mechanical stimulus, as shown in Figure $2 \mathrm{C}$. Considering an application scenario that both normal and shear stress are applied to the piezoelectric tactile sensor, the differential electric displacement is given by $d D=-d_{31} T_{1}-d_{32} T_{2}+d_{33} T_{3}$, where $d$ is the piezoelectric coefficients and $T$ is the applied stress, with the subscripts 1 and 2 describing the contribution from shear stress and 3 from the normal stress.

The piezoelectric tactile sensor has the advantages of high sensitivity, fast response time, and self-powered operation. As its operation does not have to involve the direct contact of the sensing object, it is widely used in the detection of acoustic vibration, sliding vibration, and other dynamic pressures. On the other hand, if an external force remains unchanged, the charge will be neutralized and cannot be measured statically. However, considering the unique energy collection characteristics of the piezoelectric materials, they have very important advantages in the development of fast dynamic response, low energy consumption, and selfpowered flexible tactile sensors. The piezoelectric materials commonly used in piezoelectric sensors include lead zirconate titanate $(\mathrm{PZT})^{[52-56]}, \mathrm{ZnO}^{[57]}, \mathrm{BaTiO}_{3}{ }^{[58]}, \mathrm{LiTaO}_{3}{ }^{[59,60]}$, Group III-nitride (III-N) materials (AlN and $\mathrm{GaN})^{[61-63]}$, and intrinsic flexible polyvinylidene fluoride (PVDF) ${ }^{[64-66]}$.

\section{Triboelectric mechanism}

The triboelectric effect is a common physical phenomenon in nature. When two substances with different electron binding energies come into contact with each other, one substance loses electrons to the other. This phenomenon is called the triboelectric effect ${ }^{[67]}$. Triboelectric tactile sensors are built based on the triboelectric effect. By contacting and then separating two materials with different binding energies in sequence, an electrical-potential difference is generated between the two materials. The resulting electric current flows through the external circuit and converts mechanical energy into electrical energy, as shown in Figure 2D. The research group of Wang Zhong-Lin at the Georgia Institute of Technology developed a triboelectric nanogenerator (TENG) using the triboelectric effect for the first time, which created a breakthrough way for energy collection technology ${ }^{[68]}$. As scientists continue to explore and study the triboelectric phenomenon, the triboelectric effect has been utilized for environmental energy harvesting ${ }^{[6]}$.

The triboelectric tactile sensor has the advantages of simple structure, low cost, and lightweight, thus it is an effective method for energy acquisition and dynamic information acquisition. Therefore, it is very suitable for building self-powered sensors to monitor and measure surrounding information, such as speed, 
acceleration, and displacement. The materials commonly selected for triboelectric tactile sensors include polydimethylsiloxane, polyvinyl alcohol (PVA) ${ }^{[70]}$, poly $(3,4$-ethylene dioxythiophene)-poly(styrene sulfonate) (PEDOT:PSS), polyethylene terephthalate (PET) ${ }^{[71]}$, polyacrylamide (PAM) $)^{[72]}$, and graphen $e^{[73]}$.

\section{Performance evaluation parameters}

One of the main advantages of a flexible tactile sensor over human skin is that it is a quantitative rather than a qualitative "feeling", as its electrical output signal corresponding to stimulation can be read out with an exact value. From this point of view, the performance of the flexible tactile sensor is evaluable and comparable. The main performance evaluation parameters of a flexible tactile sensor include sensitivity, sensing range, response time, repeatability, stability, etc.

The sensitivity parameters and units for tactile sensors with different mechanisms are different. Generally, the sensitivity (S) can be defined as the ratio of the increment of the output signal (M) for the tactile sensors to the increment of the input pressure $(\mathrm{P})$, described as $\mathrm{S}=\partial M / \partial P$. The sensitivity may vary with the pressure, thus exhibiting nonlinearity over the measured pressure range. For most tactile sensors, the sensitivity decreases with the increase of pressure. It is difficult to compare the sensitivity performance of tactile sensors with different mechanisms because the output physical quantities of them are different. To solve this problem, the relative change of the output signal, defined as $\Delta M / M_{0}$, can be introduced to define the sensitivity as $\mathrm{S}=\partial\left(\Delta M / M_{0}\right) / \partial P$, and the unit of the sensitivity is unified as $\mathrm{Pa}^{-1}$. In some studies, the unit of sensitivity is $\mathrm{V} \cdot \mathrm{Pa}^{-1}$ or $\mathrm{A} \cdot \mathrm{Pa}^{-1}$ for certain, piezoelectric tactile sensors, corresponding to an output signal of voltage $(\mathrm{V})$ or current $(A)$, respectively. Besides, when the input stimulus is strain, the sensitivity is unitless and is denoted as gauge factor.

The sensing range refers to the pressure range that a tactile sensor can measure normally, and it can be represented by the minimum and maximum pressures that the sensor can detect. High sensitivity tactile sensors often have a very low minimum sensing range, and the maximum sensing pressure is relatively small ${ }^{[74]}$. Meanwhile, a device that can sense a high external stimulus cannot sensitively respond to a small on $\mathrm{e}^{[75]}$. Tactile sensors with different response ranges can be applied in different situations. Pressure sensors with a large pressure detection range and low detection limit can be used for more tasks.

The response time describes how fast the tactile sensor changes its output signal in response to an external stimulus, which is also related to viscoelasticity and interface adhesion. There are different ways to calculate the response time. One is the time difference between the input time of external stimulus and the stable time of the response output, and the other is the time difference between the input time of input stimulus and the output response reach $90 \%$ or $(1-1 / \mathrm{e})$ position. Besides, the frequency response is also used to represent the response speed. Periodic dynamic pressure can be applied to the tactile sensor to obtain the dynamic response and extract the time lag between the output response and input stimulus.

Repeatability refers to the coincidence degree of response curves between different cycles of measurement under the same measurement conditions. The stability of tactile sensors is evaluated by the drift of the response values after thousands or tens of thousands of cycles. The repeatability and stability are related to the irreversible deformation of the constituent materials in the process of material pressure deformation. Generally speaking, the lower is the irreversible deformation, the better are the repeatability and stability.

In addition, flexibility is also a main evaluation parameter for the tactile sensors, and it can be realized by adopting intrinsically stretchable materials and flexible structure designs. Nowadays, most flexible tactile sensors have achieved satisfactory flexibility ${ }^{[40,44,46]}$. Tactile sensors are expected to have high sensitivity to 
detect tiny pressure and hold a high sensitivity in a broad pressure range, simultaneously, which can be achieved by further exploring functional materials and proposing novel device designs.

A perfect flexible tactile sensor that is impeccable in all sensing performance is certainly required, but most flexible tactile sensors cannot be perfect. We can design flexible tactile sensors with different performance highlights according to practical application requirements. Of course, the design of tactile sensors with an increasing number of high-level performance indicators simultaneously is an important research direction. Here, we list some typical tactile sensors with different sensing mechanisms and functional materials and compare their sensing performance including sensitivity, sensing range, response time, and cycling numbers [Table 1].

\section{DEVICE DESIGN TOWARDS THE FLEXIBLE TACTILE SENSOR SYSTEM}

In Section 2, we introduce some typical tactile sensors with outstanding sensing performance such as ultrahigh sensitivity up to $10^{7} \mathrm{kPa}^{-1}$ [Figure $3 \mathrm{~A}$ ], a fast response time of $0.03 \mathrm{~ms}$ [Figure $\left.3 \mathrm{~B}\right]^{[42]}$, broad sensing range of $10 \mathrm{~Pa}$ to $800 \mathrm{kPa}$ [Figure $3 \mathrm{C}]^{[36]}$, and good stable performance after 50,000 cycles of measurement $[\text { Figure } 3 \mathrm{D}]^{[46]}$. In other more specific applications, the corresponding performance parameter needs to be considered, including low detect limit (1.2 Pa) for response to tiny pressure $\mathrm{e}^{[76]}$, large stretchability (workable strain range of up to $187 \%$ ) for applications in flexible electronics ${ }^{[77]}$, biodegradable ${ }^{[78]}$, anisotropic ${ }^{[79]}$, recyclable $^{[80]}$, and self-powered ${ }^{[81]}$. Based on the performance constraints, the design of tactile sensors involves the choice of working principle; the device structure design including the functional material, flexible substrate, and electrode materials; and the fabrication technology. It may also entail further integration of information acquisition, processing technology, and multi-physical quantity fusion ${ }^{[82,83]}$.

\section{From single sensor to tactile sensor arrays}

A single sensor has to be expanded to sensor arrays to gain more information and adapt to the requirements of a large detection area and spatial mapping of the measurand. The adoption of a sensor array not only enables a large-area sensor but also increases the service lifetime of the sensor. Even though one or more sensor units malfunction, the overall functionality of the sensor remains. Meanwhile, information acquired from each independent single sensing unit in a sensor array can be combined for pressure mapping. For example, Kang et al. ${ }^{[84]}$ reported an $8 \times 8$ transparent capacitive sensor array with $84.6 \%$ optical transmittance, as shown in Figure 4A. The sensor array shows overall good sensing performance in the $<10 \mathrm{kPa}$ pressure range and remains stable within 200 cycles of measurement ${ }^{[84]}$. Zeng et al. ${ }^{[85]}$ developed a 4 $\times 4$ pressure sensor array based on a PDMS microstructure [Figure 4B]. The sensor array exhibits a high sensitivity of $14.268 \mathrm{kPa}^{-1}$, very low detectable pressure limit (1.5 Pa), a fast response time (<50 ms), excellent cycling stability (more than 10,000 cycles at $0.15 \mathrm{kPa}$ ), and an excellent pressure mapping ability $^{[85]}$. Gong et al. ${ }^{[86]}$ reported a $5 \times 5$ pressure sensor array based on the PDMS/ultrathin gold nanowires/PDMS sandwiching assembly with high stability (> 50,000 cycles) and demonstrated the current mapping ability under pressure, as shown in Figure 4C. Liu et al. ${ }^{[87]}$ reported a $4 \times 4$ tactile sensor array based on PZT arrays on a PDMS substrate, as shown in Figure 4D. The sensor array exhibits a high flexibility under continuous stretching $(\sim 8 \%)$, bending $\left(180^{\circ}\right.$ at a radius of $\left.3 \mathrm{~mm}\right)$, and twisting $\left(\sim 90^{\circ}\right)^{[87]}$. The rapid development of tactile sensor arrays has laid a solid foundation for realizing a large area "E-skin", mimicking the human skin's tactile functions.

\section{From single-mode sensor to multi-mode tactile sensor}

Similar to real skin, future flexible tactile sensors should be able to provide real-time feedback of various external stimuli and respond intelligently to the complex changes of the external environment. Besides simulating the basic physiological functions of human skin, such as realizing the sensing functions of 
Table 1. Summary of typical tactile sensors and comparison of their sensing performance

\begin{tabular}{|c|c|c|c|c|c|c|}
\hline Sensor mechanism & Functional materials & Sensitivity & Sensing range & Response time & Cycling numbers & Ref. \\
\hline \multirow[t]{6}{*}{ Piezo-resistive } & MWCNTS/LIG & $2.41 \mathrm{kPa}^{-1}$ & $1.2-400 \mathrm{~Pa}$ & $2 \mathrm{~ms}$ & 2000 & [35] \\
\hline & CNTs/PDMS & $\begin{array}{l}12.1 \mathrm{kPa}^{-1} \\
0.68 \mathrm{kPa}^{-1}\end{array}$ & $\begin{array}{l}600 \mathrm{~Pa} \\
1 \mathrm{kPa}\end{array}$ & $3.1 \mathrm{~ms}$ & 4000 & [34] \\
\hline & CNT & $50 \mathrm{kPa}^{-1}$ & $300 \mathrm{~Pa}-6.5 \mathrm{kPa}$ & $24 \mathrm{~ms}$ & 10000 & [29] \\
\hline & Carbon black & $5.54 \mathrm{kPa}^{-1}$ & $0.01-800 \mathrm{kPa}$ & $20 \mathrm{~ms}$ & 10000 & [36] \\
\hline & Polymer & $\begin{array}{l}7.7-41.9 \mathrm{kPa}^{-1} \\
<0.4 \mathrm{kPa}^{-1}\end{array}$ & $\begin{array}{l}<100 \mathrm{~Pa} \\
>1 \mathrm{kPa}\end{array}$ & $47 \mathrm{~ms}$ & 8000 & [41] \\
\hline & $\mathrm{MoS}_{2}$ & $0.011 \mathrm{kPa}^{-1}$ & $1-120 \mathrm{kPa}$ & $180 \mathrm{~ms}$ & 10000 & [39] \\
\hline \multirow[t]{6}{*}{ Capacitive } & PDMS/air gap & $10^{7} \mathrm{kPa}^{-1}$ & $1.5 \mathrm{kPa}$ & $0.03 \mathrm{~ms}$ & 10000 & [42] \\
\hline & $\mathrm{TiO}_{2}$ & $4.4 \mathrm{kPa}^{-1}$ & $<0.8 \mathrm{~Pa}$ & $<16 \mathrm{~ms}$ & 50000 & [46] \\
\hline & PVDF/IL & $\begin{array}{l}1.194 \mathrm{kPa}^{-1} \\
0.109 \mathrm{kPa}^{-1}\end{array}$ & $\begin{array}{l}0-0.5 \mathrm{kPa} \\
0.5-120 \mathrm{kPa}\end{array}$ & $40 \mathrm{~ms}$ & 5000 & [43] \\
\hline & 3D networks & $\begin{array}{l}0.31 \mathrm{kPa}^{-1} \\
0.03 \mathrm{kPa}^{-1}\end{array}$ & $\begin{array}{l}0.05-3.8 \mathrm{kPa} \\
3.8-6.3 \mathrm{kPa}\end{array}$ & - & 5000 & [51] \\
\hline & Ionic conductor & $54.31 \mathrm{kPa}^{-1}$ & $<0.5 \mathrm{kPa}$ & $29 \mathrm{~ms}$ & 5400 & [47] \\
\hline & $\mathrm{PVA} / \mathrm{H}_{3} \mathrm{PO}_{4}$ & $>220 \mathrm{kPa}^{-1}$ & $0.08 \mathrm{~Pa}-360 \mathrm{kPa}$ & $9 \mathrm{~ms}$ & 5000 & [44] \\
\hline \multirow[t]{6}{*}{ Piezo-electric } & $\mathrm{PZT}$ & $\sim 5 \mu \mathrm{A} \cdot \mathrm{kPa}^{-1}$ & $10 \mathrm{~Pa}$ & $\sim 0.1 \mathrm{~ms}$ & - & [52] \\
\hline & $\mathrm{PZT}$ & $0.018 \mathrm{~V} \cdot \mathrm{kPa}^{-1}$ & $1-30 \mathrm{kPa}$ & $60 \mathrm{~ms}$ & 5000 & [53] \\
\hline & $\mathrm{PVDF} / \mathrm{ZnO}$ & $0.33 \mathrm{~V} \cdot \mathrm{kPa}^{-1}$ & $9 \mathrm{~N}$ & $16 \mathrm{~ms}$ & 5000 & [57] \\
\hline & $\begin{array}{l}\text { PDA@BTO/ } \\
\text { PVDF }\end{array}$ & $9.3 \mathrm{~V} \cdot \mathrm{kPa}^{-1}$ & $33 \mathrm{kPa}$ & $61 \mathrm{~ms}$ & 1000 & [58] \\
\hline & BTO/PVDF & $3.95 \mathrm{~V} \cdot \mathrm{N}^{-1}$ & $3 N$ & - & 7400 & {$[65]$} \\
\hline & $\mathrm{PVDF} / \mathrm{SnO}_{2} \mathrm{NS} @ \mathrm{SiO}_{2}$ & $0.99 \mathrm{~V} \cdot \mathrm{kPa}^{-1}$ & $10-30 \mathrm{kPa}$ & $1 \mathrm{~ms}$ & - & [64] \\
\hline \multirow[t]{4}{*}{ Tribo-electric } & PDMS/PVA NFs & - & >100\% (strain) & $70 \mathrm{~ms}$ & 12000 & [70] \\
\hline & PDMS/PET & $0.06 \mathrm{kPa}^{-1}$ & $150 \mathrm{kPa}$ & $70 \mathrm{~ms}$ & 10000 & [71] \\
\hline & PAM/PEDOT:PSS & 1.58 & $2850 \%$ (strain) & $200 \mathrm{~ms}$ & 1200 & [72] \\
\hline & PEN/Graphene & $\begin{array}{l}1.63 \mathrm{kPa}^{-1} \\
0.04 \mathrm{kPa}^{-1}\end{array}$ & $\begin{array}{l}0.1-6 \mathrm{kPa} \\
6-100 \mathrm{kPa}\end{array}$ & $<3 \mathrm{~ms}$ & 10000 & [73] \\
\hline
\end{tabular}

pressure, strain, temperature, and humidity, other characteristics of the intelligent tactile sensor should be explored, including the detection of sound, light, heat, magnetic field, and chemical information. In this situation, a single-mode sensor cannot fulfill all of the requirements, and researchers have developed dualmode or even multi-mode sensors to realize various sensing functions. For example, Qiu et al. ${ }^{[8]}$ reported a dual-mode sensor based on interlocked piezoresistive and piezoelectric films, realizing the complementary performance of the two sensing mechanisms, and thus improving the sensitivity and broadening the sensing range [Figure 5A]. Hwang et al. ${ }^{[89]}$ reported a transparent sensor combining piezoresistive and capacitive transduction mechanisms, which can distinguish touch and pressure, as shown in Figure 5B. Besides the direct combination of two sensing mechanisms in a single device design, a single sensor can respond to a variety of stimuli by selecting specific functional materials. For example, Yang et al. ${ }^{\left[{ }^{[0]}\right.}$ reported a selfpowered elastic conductor based on PEDOT:PSS natural rubber films and realized both temperature- and tensile strain-sensing abilities, as shown in Figure 5C. You et al. ${ }^{[91]}$ presented multimodal ion-electronic skin based on a deformable artificial ionic receptor, which can differentiate thermal and mechanical information without signal interference, as shown in Figure 5D. Wang et al. ${ }^{[21]}$ used the blow-spinning method to prepare integrated wearable electronics based on $3 \mathrm{D}$ inorganic nanofiber IGZO networks, which can detect and differentiate multiple stimuli including test gas, strain, temperature, light, body movement, and respiratory functions [Figure 5E]. 
A

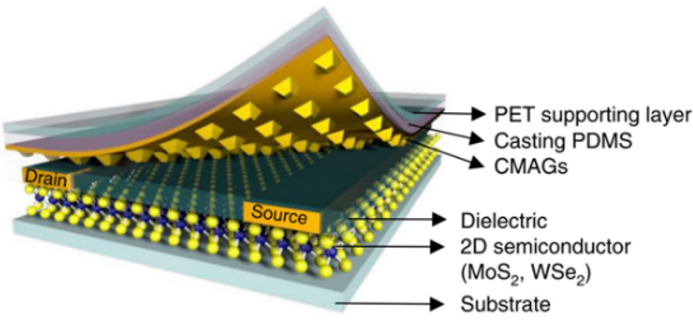

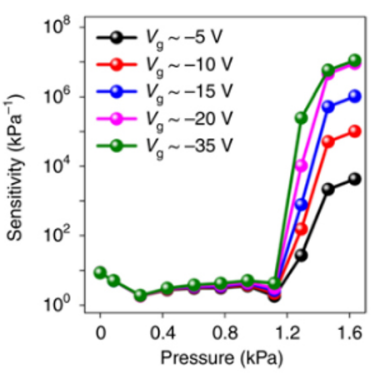

B

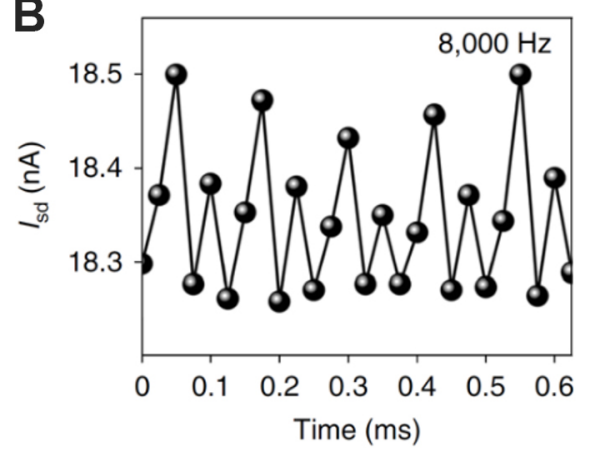

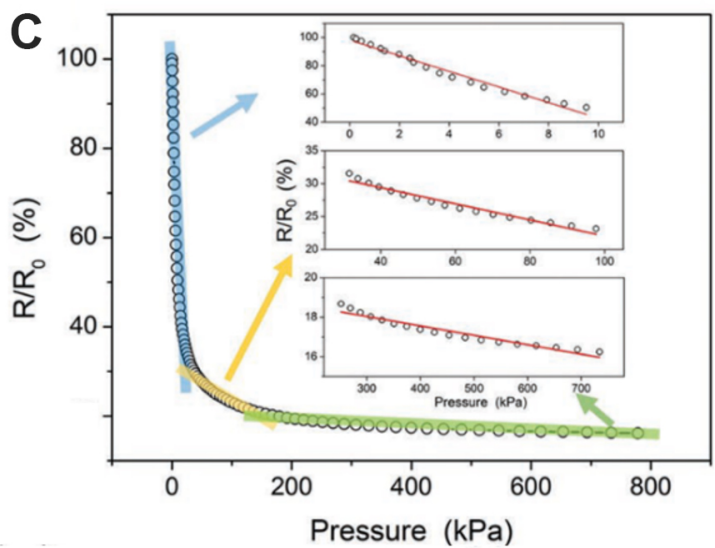

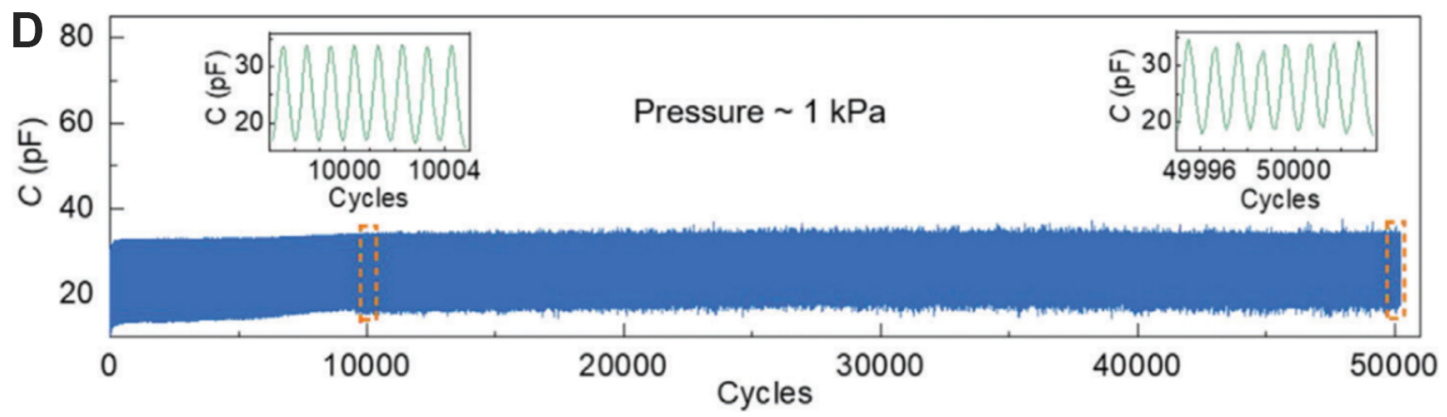

Figure 3. Typical tactile sensors with outstanding sensing performance. (A) Schematic of the tactile sensor based on air gaps and $\mathrm{MoS}_{2}$ transistor and corresponding sensitivity up to $10^{7} \mathrm{kPa}^{-1}$. (B) Highlighting the rapid response time, $0.03125 \mathrm{~ms}$. (A, B) Reproduced with permission from Ref. ${ }^{[42]}$. Copyright ${ }^{\odot}$ 2020. Springer Nature. (C) Broad sensing range (10 Pa-800 kPa). Reproduced with permission from Ref. ${ }^{[36]}$. Copyright ${ }^{\complement}$ 2018. John Wiley and Sons. (D) Stable performance after 50,000 cycles of measurement. Reproduced with permission from Ref. ${ }^{[46]}$. Copyright ${ }^{\circ}$ 2020. John Wiley and Sons.

\section{Tactile sensors integrated with transistors}

The small output signal of a tactile sensor has to be filtered and amplified for information interpretation. However, many demonstrations usually connect the sensor to high-precision equipment to demonstrate the functionality of the sensor, which is not practical in real-life applications. The interconnections required to connect the sensor with the processing circuit bring extra noise as well as increase the overall area. To alleviate this problem, researchers have explored novel device structures to directly integrate tactile sensors with transistors. For example, Huang et al. ${ }^{[42]}$ reported an ultra-high sensitivity capacitive sensor based on the vertical integration of air-gap gates (capacitor sensor) with a two-dimensional semiconductor transistor, as shown in Figure 3A. The integrated 2D transistor is used to amplify the output signal, resulting in an ultra-high sensitivity up to $10^{7} \mathrm{kPa}^{-1}$, compared to $44 \mathrm{kPa}^{-1}$ for the traditional air-gap pressure sensor ${ }^{[42]}$. Wang et al. ${ }^{[92]}$ reported a piezoelectric tactile sensor integrated with an organic field-effect transistor, as shown in Figure 6A. External mechanical force is converted into a piezoelectric voltage to drive the transistor, and the piezoelectric voltage can be effectively amplified by the transistors to improve the 
A
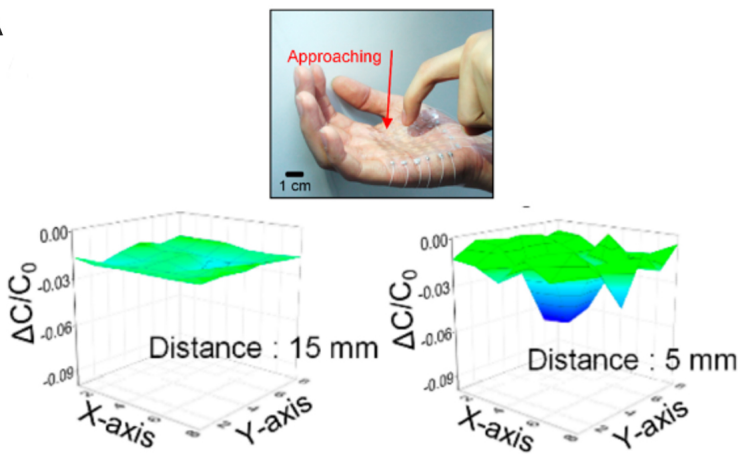

B

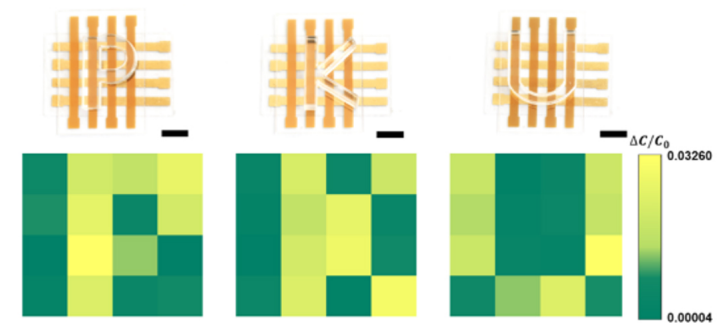

C

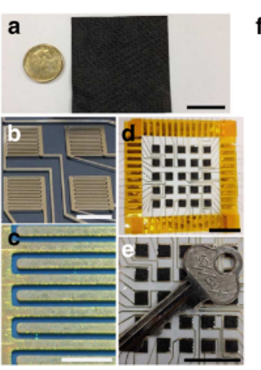

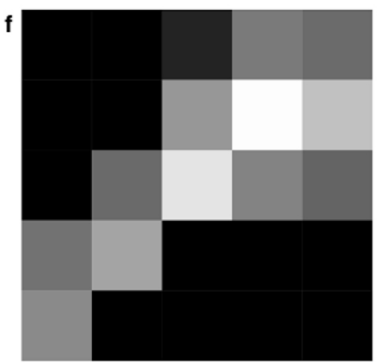
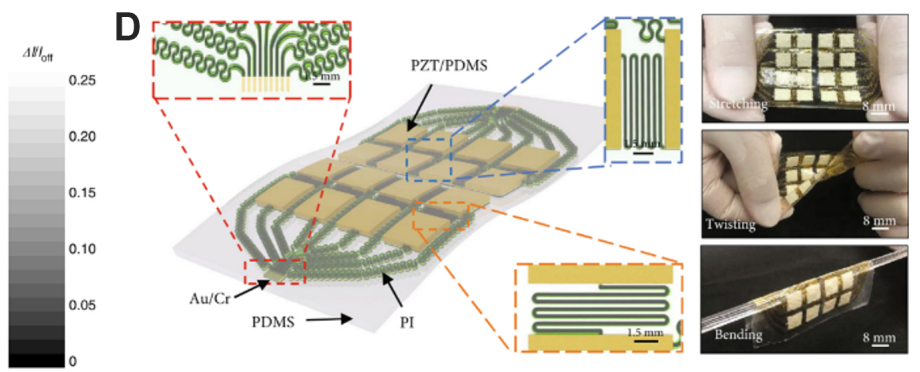

Figure 4. Tactile sensor arrays. (A) Image of an $8 \times 8$ transparent capacitive sensor array on the palm and the capacitance changes when the finger approaches at 15 and $5 \mathrm{~mm}$. Reproduced with permission from Ref. ${ }^{[84]}$. Copyright ${ }^{\odot} 2017$. American Chemical Society. (B) Pressure mapping on a $4 \times 4$ pressure sensor array by placing acrylic boards of " $P$ ", " $K$ ", and " $U$ ". Reproduced with permission from Ref. ${ }^{[85]}$. Copyright ${ }^{\circ}$ 2019. American Chemical Society. (C) (a-c) Images of a $5 \times 5$ tactile sensor array. (d-f) The current mapping ability under the pressure of a key. Reproduced with permission from Ref. ${ }^{[86]}$. Copyright ${ }^{\odot} 2014$. Springer Nature. (D) Images of a $4 \times 4$ tactile sensor array and a highly flexible ability. Reproduced with permission from Ref. ${ }^{[87]}$. Copyright ${ }^{\odot} 2020$. OAE Publishing Inc.

sensitivity of the sensor. In addition, a $3 \times 3$ integrated sensor array was fabricated on PET substrate, showing a good stability and flexible performance, as shown in Figure $6 \mathrm{~B}^{[92]}$. Dagdeviren et al. ${ }^{[52]}$ reported an $8 \times 8$ PZT piezoelectric sensor array, in which the total signal output is horizontally integrated with a metal oxide semiconductor field-effect transistor that transforms the output voltage signal into the drain current [Figure $6 \mathrm{C}$ ]. Oh et al. ${ }^{[93]}$ reported scalable tactile sensor arrays based on dual-gate piezoelectric zinc oxide thin-film transistors, which can detect compressive and shear stress, as shown in Figure 6D. This ability has application value in achieving the slip and grip function of closed-loop robotics ${ }^{[93]}$. Guo et al. ${ }^{[94]}$ reported an integrated contact lens sensor system, integrating temperature sensor, glucose sensor, photodetector, and multiple $\mathrm{MoS}_{2}$ transistors on the same platform, as shown in Figure 6E, providing a possibility for realizing a multi-functional sensor system. Triboelectric sensors, as promising self-powered sensors, can also be integrated with transistors, showing the feasibility of novel self-powered sensor systems ${ }^{[95]}$. For example, Zhao et al. ${ }^{[96]}$ reported a triboelectric sensor integrated with a stretchable organic transistor based on PDMS substrate for smart tactile interaction [Figure $6 \mathrm{~F}$ ].

\section{APPLICATIONS}

With the rapid performance improvement of tactile sensors and sensor arrays, various emerging application scenarios have been demonstrated in the fields of artificial intelligence, human-machine interaction, virtual and augmented reality, and healthcare systems. For example, the tactile sensor system in a humanoid robot plays an important role in evaluating the overall performance of the robot ${ }^{[97]}$. The tactile sensor system is an indispensable part of artificial arms and knee joints, flexible exoskeletons, intelligent prostheses, and medical robotics. Quantification of the pressure on the joint during moving extraordinarily relies upon the feedback signal from the tactile sensor system ${ }^{[98-100]}$. A flexible piezoelectric sensor array based on the 
A
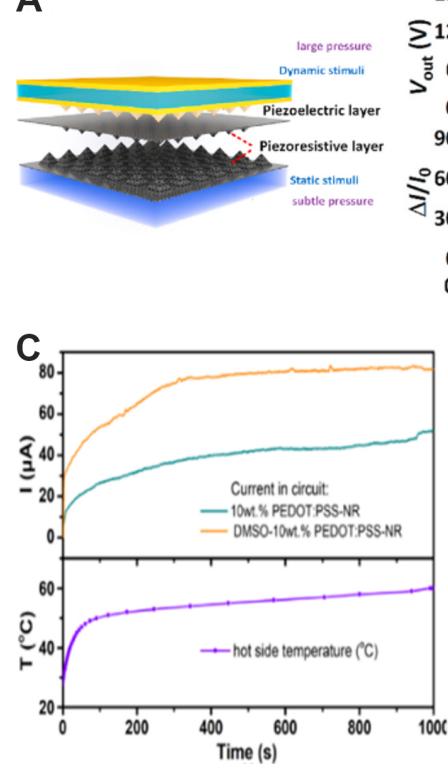
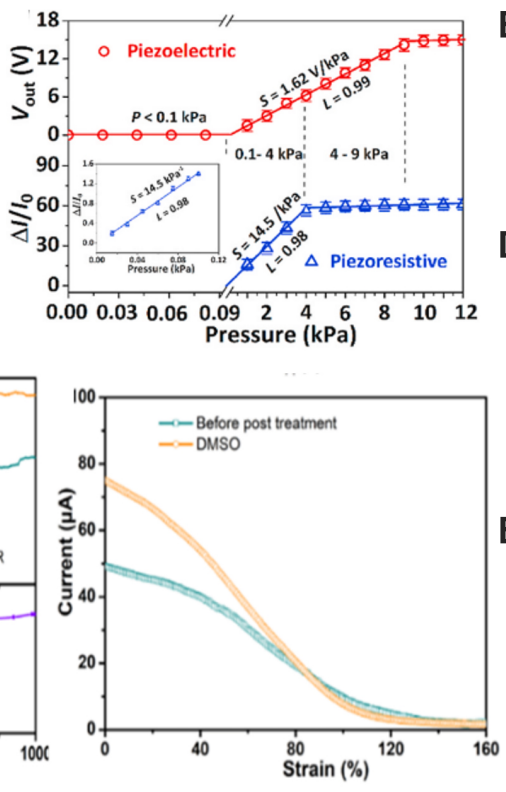

B
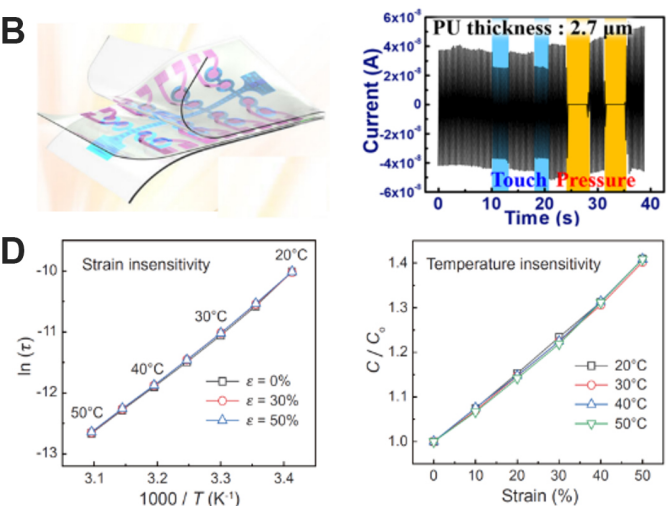

E

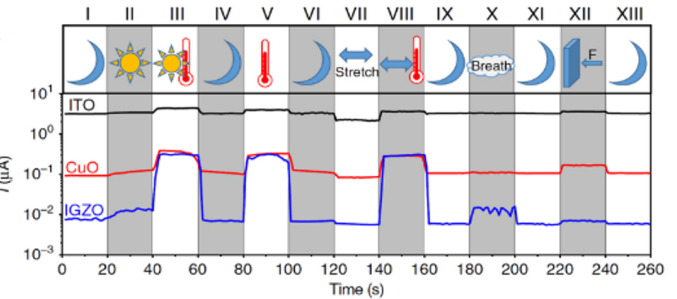

Figure 5. Multi-mode tactile sensors. (A) Schematic illustrations of the structure and sensitivity (sensing range 0-12 kPa) of a dual-mode sensor. Reproduced with permission from Ref. ${ }^{[88]}$. Copyright ${ }^{\circ}$ 2020. Elsevier. (B) Schematic diagram and current versus time of the transparent dual-mode sensor, which can distinguish touch and pressure. Reproduced with permission from Ref. ${ }^{[89]}$. Copyright ${ }^{\circ} 2019$. Springer Nature. (C) The current response to temperature and strain stimulus respectively. Reproduced with permission from Ref. ${ }^{[90]}$. Copyright $^{\mathbb{C}}$ 2021. American Chemical Society. (D) Characteristics of multimodal sensor and its responses to heating and stretching without signal interference. Reproduced with permission from Ref. ${ }^{[91]}$. Copyright ${ }^{\circ}$ 2020. The American Association for the Advancement of Science. (E) Multiple stimulus responses including test strain, temperature, light, body movement, and respiratory functions. Reproduced with permission from Ref. ${ }^{[21]}$. Copyright ${ }^{\odot}$ 2020. Springer Nature.

inorganic AlN thin film was attached to the skin, and the subtle facial strain was decoded by taking advantage of machine learning algorithms and the mapped data from the sensor arrays ${ }^{[25]}$. Apart from the application in common robots, tactile sensor systems also have great potential application value in micro/nanorobots for a variety of deep-sea exploration and biomedical applications ${ }^{[101,102]}$. In virtual and augmented reality field, tactile sensors are also widely used. For example, a tactile sensor system used for real-time detection of eyeball vergence in virtual reality can treat the astigmatism of eyes at home ${ }^{[103]}$. Using a skin-integrated wireless haptic interface, people can touch far-away relatives [Figure 7A]. Those with disabled upper limbs can regain the sense of touch with the help of a flexible sensor system [Figure 7B]. People who play fighting games may feel the virtual pain from the game [Figure $7 \mathrm{C}]^{[81]}$. In addition, a soft virtual reality glove integrating a pneumatic actuator with a piezoelectric tactile sensor can transmit the real stimulus to the users from virtual reality [Figure $7 \mathrm{D}]^{[104]}$.

Recently, tactile sensors have been widely used in medical treatment, health monitoring, and for collecting and testing the physiological indexes including blood pressure, glucose, pulse, temperature, respiratory rate, voice, electrocardiogram, electromyogram, and sleep status ${ }^{[28,105-109]}$. For instance, an integrated wireless tactile sensor system was used for health monitoring in neonatal patients and has already entered into service in hospitals ${ }^{[23]}$. Pulse wave detection is a common application function of wearable sensors and can be realized with a self-powered microstructured piezoresistive tactile sensor [Figure $8 \mathrm{~A}]^{[110]}$. Similarly, the blood pressure monitoring is enabled with a conformal piezoelectric sensor [Figure 8B] ${ }^{[111]}$. A piezoelectric sensor based on PZT ribbons was developed for energy harvesting from heartbeat and pulmonary respiration, which could offer sufficient power supply for the operation of heart pacemakers without an external battery ${ }^{[54]}$. Multifunctional electronic garments based on silica nanoparticle/PDMS layer showed 
A

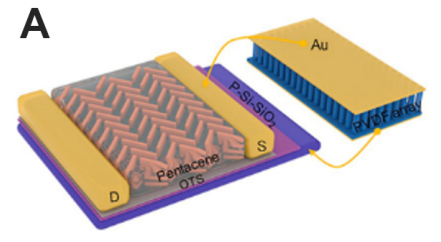

C

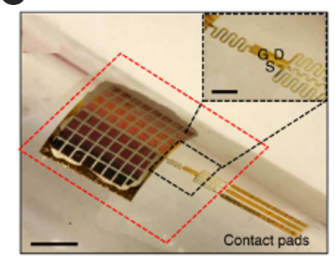

E Lens

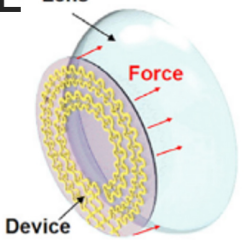

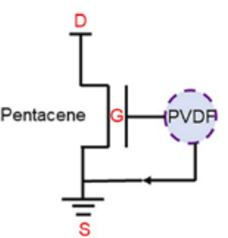

B

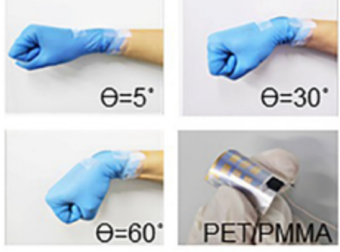

D
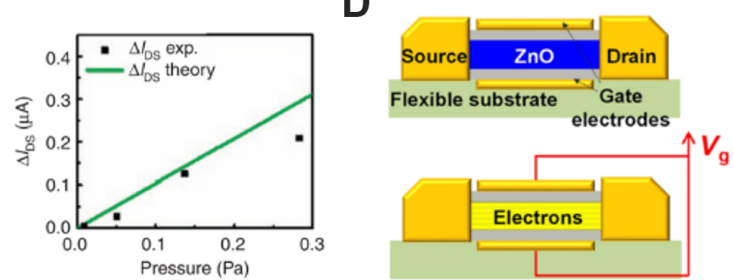

$\mathbf{F}$

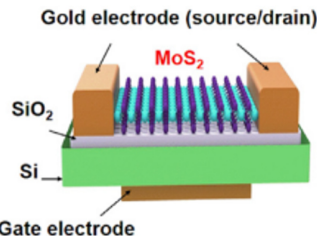

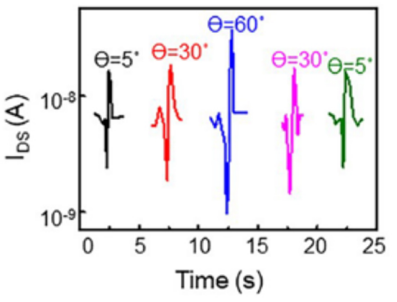
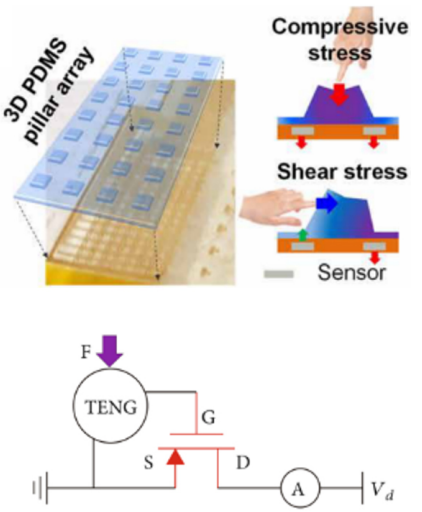

Figure 6. Tactile sensors integrated with transistors. (A) Schematic of the composition and mechanism of the tactile sensor integrated with a field-effect transistor. (B) The image and results of the flexible performance measurement for the system in (A). ( $A, B$ ) Reproduced with permission from Ref. ${ }^{[92]}$. Copyright ${ }^{\odot} 2020$. Elsevier. (C) Photograph and the drain current response to the pressure of the sensor array integrated with a field-effect transistor. Reproduced with permission from Ref. ${ }^{[52]}$. Copyright ${ }^{\circ}$ 2014. Springer Nature. (D) Schematic of the thin-film transistor and independent detection capability of compressive and shear stress using the threedimensional PDMS pillar array. Reproduced with permission from Ref. ${ }^{[93]}$. Copyright ${ }^{\odot} 2020$. OAE Publishing Inc. (E) Illustration of the sensor layer and 3D schematics of $\mathrm{MoS}_{2}$ transistor. Reproduced with permission from Ref. ${ }^{[94]}$. Copyright ${ }^{\odot}$ 2021. Elsevier. (F) Working principle and equivalent circuit of tactile sensor integrated with a transistor. Reproduced with permission from Ref. ${ }^{[96]}$. Copyright ${ }^{\complement} 2020$. OAE Publishing Inc.

waterproof, breathable, and antibacterial performance and enabled the practical application in wearable electronics ${ }^{[112]}$. Infrared electronic skin for the promotion of cutaneous wound healing was reported, as shown in Figure $8 \mathrm{C}^{[113]}$, bringing active therapeutic purpose and clinical value. By integrating sensors, Bluetooth, a cloud data infrastructure, automatic data processing platform, and a user interface, a health monitoring system can detect the frequency and intensity of coughs and their changes with the state of COVID-19 disease progression. In the context of the global COVID-19 pandemic, this ability to continuously monitor key physiological parameters of the disease is very important ${ }^{[114]}$.

\section{CONCLUSIONS AND PERSPECTIVES}

In this review, we briefly summarize the recent progress of flexible tactile sensor systems, including the sensing mechanisms, the common functional materials, the important parameters to evaluate the performance, the device design, and the main applications. In particular, the development trend of tactile sensor systems is discussed. Besides the performance optimization for a single tactile sensor emphasizing certain performance indicators, the tactile sensor array for large-area pressure detection and the multi-mode tactile sensor aiming to obtain multiple sensing capabilities and tactile sensors integrated with transistors will be the development trends of integrated tactile sensor systems to satisfy the requirements of various applications in virtual and augmented reality, intelligent robots, human-machine interfaces, medical treatment, and wearable health monitoring. 
A

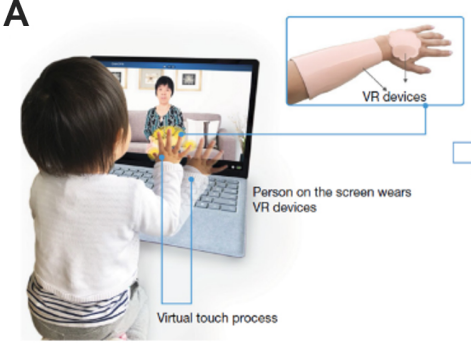

C

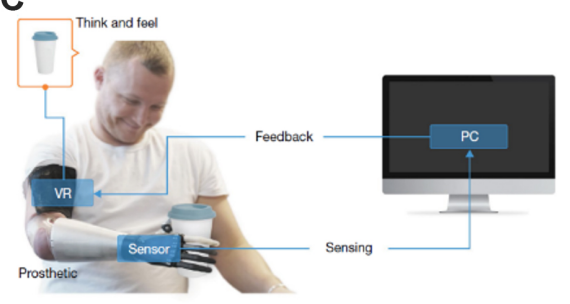

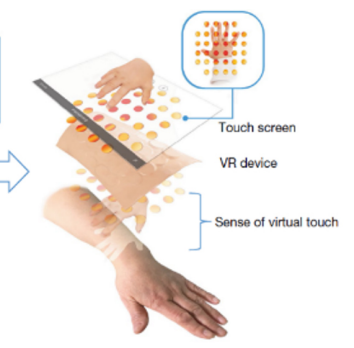

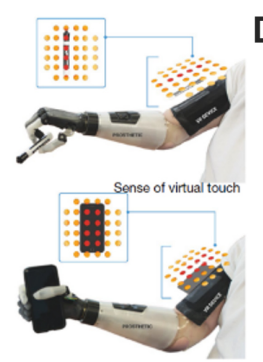

B
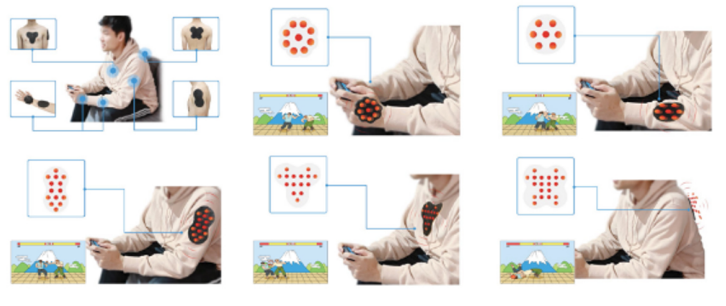

D
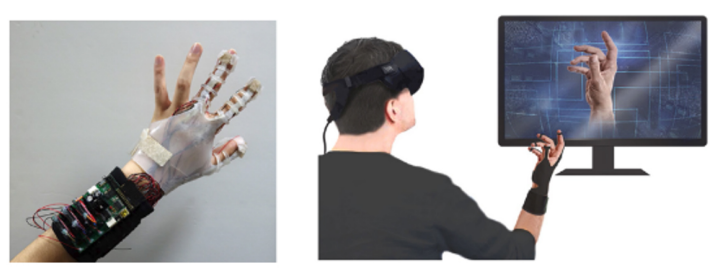

Figure 7. Applications in virtual and augmented reality of tactile sensing systems. (A) The child touches grandmother on the screen and the grandmother wearing the VR devices on arm can feel the touch. (B) The man with disabled upper limbs has the sense of virtual touch with the help of the sensor system. (C) The man playing fighting games feels the virtual pain from the game. (A-C) Reproduced with permission from Ref. ${ }^{[81]}$. Copyright ${ }^{\circ}$ 2019. Springer Nature. (D) The actual appearance of the virtual reality glove, and the man wearing the virtual reality glove can feel the real stimulus from the virtual reality. Reproduced with permission from Ref. ${ }^{[104]}$. Copyright ${ }^{\odot} 2019$. Springer Nature.

A
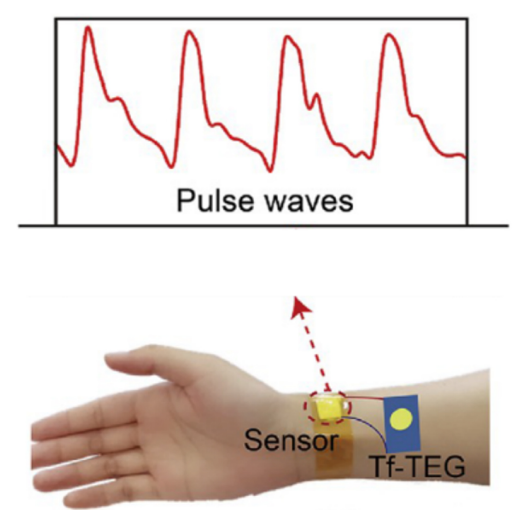

B
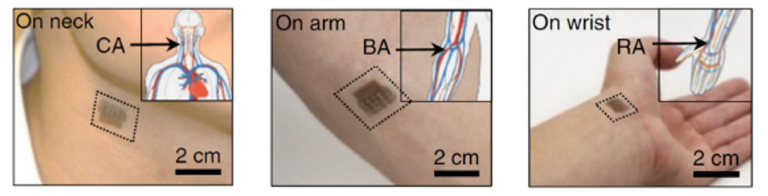

C
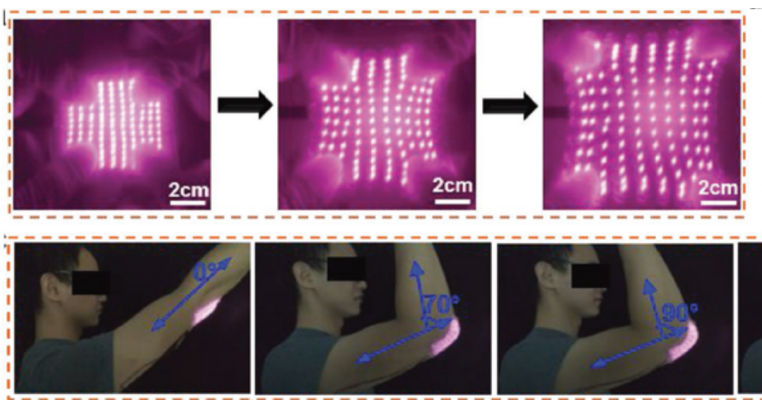
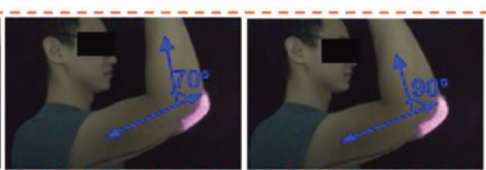
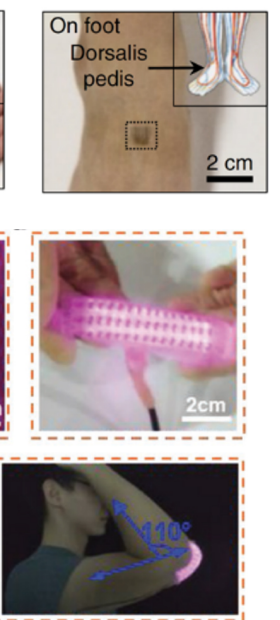

Figure 8. Applications in medical treatment and health monitoring. (A) Pulse waves detected by a self-powered sensor. Reproduced with permission from Ref. ${ }^{[110]}$. Copyright ${ }^{\odot}$ 2020. Elsevier. (B) Measurement of blood pressure in different parts of a body using a tactile sensor. Reproduced with permission from Ref. ${ }^{[11]}$. Copyright ${ }^{\odot}$ 2018. Springer Nature. (C) Infrared electronic skin with different elbow movement. Reproduced with permission from Ref. ${ }^{[113]}$. Copyright ${ }^{\oplus}$ 2019. John Wiley and Sons.

The device performance of the tactile sensor remains a key challenge for realizing advanced tactile sensing systems. Highly sensitive tactile sensors with a broad pressure range are always a research focus. At the same time, reducing detection area, improving detecting accuracy, and distinguishing the stimulations of various force ${ }^{[115]}$ coming from different directions are also challenging for advanced sensing systems. For the multimode tactile sensor, a critical problem is how to solve the crosstalk when more than two physical parameters are changing together, especially the changing of temperature, which can easily influence the other physical parameters. In the aspect of device design towards flexible tactile sensor systems, the 
realization of scalable sensor arrays is the foundation of large area tactile sensor systems.

To satisfy the requirements of various applications in virtual and augmented reality, intelligent robots, human-machine interfaces, medical treatment, and wearable health monitoring, the future trend of the flexible tactile sensor system is summarized as follows. First, besides the basic requirement of flexibility and conformality, an ideal flexible tactile sensor is expected to exhibit a high sensitivity and multi-function integration to realize multi-dimensional synchronous sensing. Second, recent strategies toward low-power or even self-powered tactile sensors could overcome the energy constraints for longtime monitoring in wearable electronics. Third, various machine learning algorithms are adopted to interpret or enhance the information acquired from the tactile sensor array, so that the artificial intelligence auxiliary detection for human activity monitoring, medical services, human-machine interfaces, artificial intelligence robots, and other applications can be realized. Similar to real skin, future flexible tactile sensors are expected to provide real-time feedback of various external stimuli and respond intelligently to the complex changes of the external environment. In addition, besides simulating the basic physiological functions of human skin, such as the sensing functions of pressure, strain, temperature, and humidity, and obtaining quantitative data, other characteristics of the intelligent tactile sensors should also be explored, including the detection of sound, light, magnetic field, and chemical information. Moreover, emerging smart contact lenses based on integrated flexible tactile sensor systems are also expected for virtual and augmented reality applications. Therefore, research on flexible tactile sensors and the integration systems is an important subject in the science community and its practical applications in human activity monitoring, medical services, humanmachine interfaces, and artificial intelligence robots will promote the development of human civilization.

\section{DECLARATIONS}

\section{Authors' contributions}

Writing - original draft: Zhu J

Writing - review \& editing: Zhou C

Supervision, writing - review \& editing, project administration: Zhang M

\section{Availability of data and materials}

Not applicable.

\section{Financial support and sponsorship}

This work was supported by Shenzhen Fundamental Research Program (No. GXWD2020123116580700720200827122756001), Guangdong Science and Technology Plan Project 2019A050510011, and National Natural Science Foundation of China 62074008.

\section{Conflicts of interest}

All authors declared that there are no conflicts of interest.

\section{Ethical approval and consent to participate}

Not applicable.

\section{Consent for publication}

Not applicable.

\section{Copyright}

(c) The Author(s) 2021. 


\section{REFERENCES}

1. Zhu M, He T, Lee C. Technologies toward next generation human machine interfaces: From machine learning enhanced tactile sensing to neuromorphic sensory systems. Applied Physics Reviews 2020;7:031305. DOI

2. Dahiya RS, Mittendorfer P, Valle M, Cheng G, Lumelsky VJ. Directions toward effective utilization of tactile skin: a review. IEEE Sensors $J$ 2013;13:4121-38. DOI

3. Wang D, Wang L, Shen G. Nanofiber/nanowires-based flexible and stretchable sensors. J Semicond 2020;41:041605. DOI

4. Takei K, Gao W, Wang C, Javey A. Physical and chemical sensing with electronic skin. Proc IEEE 2019;107:2155-67. DOI

5. Qi D, Zhang K, Tian G, Jiang B, Huang Y. Stretchable electronics based on PDMS substrates. Adv Mater 2021;33:e2003155. DOI PubMed

6. Ma Z, Kong D, Pan L, Bao Z. Skin-inspired electronics: emerging semiconductor devices and systems. J Semicond 2020;41:041601. DOI

7. Li J, Bao R, Tao J, Peng Y, Pan C. Recent progress in flexible pressure sensor arrays: from design to applications. J Mater Chem C 2018;6:11878-92. DOI

8. Li D, Yao K, Gao Z, Liu Y, Yu X; Department of Biomedical Engineering; City University of Hong Kong; Hong Kong; China. Recent progress of skin-integrated electronics for intelligent sensing. Light: Advanced Manufacturing 2021;2:39-58. DOI

9. Kumar S, Pavelyev V, Tripathi N, et al. Review - recent advances in the development of carbon nanotubes based flexible sensors. $J$ Electrochem Soc 2020;167:047506. DOI

10. Kim JJ, Wang Y, Wang H, Lee S, Yokota T, Someya T. Skin electronics: next-generation device platform for virtual and augmented reality. Adv Funct Mater 2021. DOI

11. Jiang C, Cheng X, Nathan A. Flexible ultralow-power sensor interfaces for E-skin. Proc IEEE 2019;107:2084-105. DOI

12. Jeon S, Lim S, Trung TQ, Jung M, Lee N. Flexible multimodal sensors for electronic skin: principle, materials, device, array architecture, and data acquisition method. Proc IEEE 2019;107:2065-83. DOI

13. Heo JS, Hossain MF, Kim I. Challenges in design and fabrication of flexible/stretchable carbon- and textile-based wearable sensors for health monitoring: a critical review. Sensors (Basel) 2020;20:3927. DOI PubMed PMC

14. Cheng G, Dean-leon E, Bergner F, Rogelio Guadarrama Olvera J, Leboutet Q, Mittendorfer P. A comprehensive realization of robot skin: sensors, sensing, control, and applications. Proc IEEE 2019;107:2034-51. DOI

15. Chen W, Yan X. Progress in achieving high-performance piezoresistive and capacitive flexible pressure sensors: A review. $J$ Mater Sci Mater Med 2020;43:175-88. DOI

16. Bariya M, Nyein HYY, Javey A. Wearable sweat sensors. Nat Electron 2018;1:160-71. DOI

17. Miao P, Wang J, Zhang C, Sun M, Cheng S, Liu H. Graphene nanostructure-based tactile sensors for electronic skin applications. Nanomicro Lett 2019;11:71. DOI PubMed PMC

18. Wan Y, Wang Y, Guo CF. Recent progresses on flexible tactile sensors. Materials Today Physics 2017;1:61-73. DOI

19. Huang S, Liu Y, Zhao Y, Ren Z, Guo CF. Flexible electronics: stretchable electrodes and their future. Adv Funct Mater 2019;29:1805924. DOI

20. Lee S, Franklin S, Hassani FA, et al. Nanomesh pressure sensor for monitoring finger manipulation without sensory interference. Science 2020;370:966-70. DOI PubMed

21. Wang B, Thukral A, Xie Z, et al. Flexible and stretchable metal oxide nanofiber networks for multimodal and monolithically integrated wearable electronics. Nat Commun 2020;11:2405. DOI PubMed PMC

22. Park Y, Kwon K, Kwak SS, et al. Wireless, skin-interfaced sensors for compression therapy. Sci Adv 2020;6:eabe1655. DOI PubMed PMC

23. Chung HU, Rwei AY, Hourlier-Fargette A, et al. Skin-interfaced biosensors for advanced wireless physiological monitoring in neonatal and pediatric intensive-care units. Nat Med 2020;26:418-29. DOI PubMed PMC

24. Kwak JW, Han M, Xie Z, et al. Wireless sensors for continuous, multimodal measurements at the skin interface with lower limb prostheses. Sci Transl Med 2020;12:eabc4327. DOI PubMed PMC

25. Sun T, Tasnim F, McIntosh RT, et al. Decoding of facial strains via conformable piezoelectric interfaces. Nat Biomed Eng 2020;4:954-72. DOI PubMed

26. Tai LC, Ahn CH, Nyein HYY, et al. Nicotine monitoring with a wearable sweat band. ACS Sens 2020;5:1831-7. DOI PubMed

27. Lin Y, Bariya M, Javey A. Wearable Biosensors for Body Computing. Adv Funct Mater 2020. DOI

28. Liu Q, Liu Y, Wu F, et al. Highly sensitive and wearable $\mathrm{In}_{2} \mathrm{O}_{3}$ nanoribbon transistor biosensors with integrated on-chip gate for glucose monitoring in body fluids. ACS Nano 2018;12:1170-8. DOI PubMed

29. Xie C, Zhang M, Du W, et al. Sensing-range-tunable pressure sensors realized by self-patterned-spacer design and vertical CNT arrays embedded in PDMS. RSC Adv 2020;10:33558-65. DOI

30. Park J, Lee Y, Hong J, et al. Giant tunneling piezoresistance of composite elastomers with interlocked microdome arrays for ultrasensitive and multimodal electronic skins. ACS Nano 2014;8:4689-97. DOI PubMed

31. Bae GY, Pak SW, Kim D, et al. Linearly and highly pressure-sensitive electronic skin based on a bioinspired hierarchical structural array. Adv Mater 2016;28:5300-6. DOI PubMed

32. Won SM, Wang H, Kim BH, et al. Multimodal sensing with a three-dimensional piezoresistive structure. ACS Nano 2019;13:109729. DOI PubMed

33. Zhu P, Wang Y, Wang Y, Mao H, Zhang Q, Deng Y. Flexible 3D architectured piezo/thermoelectric bimodal tactile sensor array for E-skin application. Adv Energy Mater 2020;10:2001945. DOI 
34. Sun X, Sun J, Li T, et al. Flexible tactile electronic skin sensor with 3D force detection based on porous CNTs/PDMS nanocomposites. Nanomicro Lett 2019;11:57. DOI PubMed PMC

35. Zhao J, Luo J, Zhou Z, et al. Novel multi-walled carbon nanotubes-embedded laser-induced graphene in crosslinked architecture for highly responsive asymmetric pressure sensor. Sensors and Actuators A: Physical 2021;323:112658. DOI

36. Wang Z, Guan X, Huang H, Wang H, Lin W, Peng Z. Full 3D printing of stretchable piezoresistive sensor with hierarchical porosity and multimodulus architecture. Adv Funct Mater 2019;29:1807569. DOI

37. Zhao J, He C, Yang R, et al. Ultra-sensitive strain sensors based on piezoresistive nanographene films. Appl Phys Lett 2012;101:063112. DOI

38. Qiu L, Bulut Coskun M, Tang Y, et al. Ultrafast dynamic piezoresistive response of graphene-based cellular elastomers. Adv Mater 2016;28:194-200. DOI PubMed

39. Park YJ, Sharma BK, Shinde SM, et al. All $\mathrm{MoS}_{2}$-based large area, skin-attachable active-matrix tactile sensor. ACS Nano 2019;13:3023-30. DOI PubMed

40. Park J, Lee Y, Hong J, et al. Tactile-direction-sensitive and stretchable electronic skins based on human-skin-inspired interlocked microstructures. ACS Nano 2014;8:12020-9. DOI PubMed

41. Pan L, Chortos A, Yu G, et al. An ultra-sensitive resistive pressure sensor based on hollow-sphere microstructure induced elasticity in conducting polymer film. Nat Commun 2014;5:3002. DOI PubMed

42. Huang Y, Liu Y, Ma C, et al. Sensitive pressure sensors based on conductive microstructured air-gap gates and two-dimensional semiconductor transistors. Nat Electron 2020;3:59-69. DOI

43. Liu Q, Liu Z, Li C, et al. Highly transparent and flexible iontronic pressure sensors based on an opaque to transparent transition. $A d v$ Sci (Weinh) 2020;7:2000348. DOI PubMed PMC

44. Bai N, Wang L, Wang Q, et al. Graded intrafillable architecture-based iontronic pressure sensor with ultra-broad-range high sensitivity. Nat Commun 2020;11:209. DOI PubMed PMC

45. Yang X, Chen S, Shi Y, Fu Z, Zhou B. A flexible highly sensitive capacitive pressure sensor. Sensors and Actuators A: Physical 2021;324:112629. DOI

46. Fu M, Zhang J, Jin Y, Zhao Y, Huang S, Guo CF. A highly sensitive, reliable, and high-temperature-resistant flexible pressure sensor based on ceramic nanofibers. Adv Sci (Weinh) 2020;7:2000258. DOI PubMed PMC

47. Qiu Z, Wan Y, Zhou W, et al. Ionic skin with biomimetic dielectric layer templated from calathea zebrine leaf. Adv Funct Mater 2018;28:1802343. DOI

48. Sarwar MS, Dobashi Y, Preston C, Wyss JK, Mirabbasi S, Madden JD. Bend, stretch, and touch: locating a finger on an actively deformed transparent sensor array. Sci Adv 2017;3:e1602200. DOI PubMed PMC

49. Tee BC, Chortos A, Dunn RR, Schwartz G, Eason E, Bao Z. Tunable flexible pressure sensors using microstructured elastomer geometries for intuitive electronics. Adv Funct Mater 2014;24:5427-34. DOI

50. Navaraj W, Dahiya R. Fingerprint-enhanced capacitive-piezoelectric flexible sensing skin to discriminate static and dynamic tactile stimuli. Advanced Intelligent Systems 2019;1:1900051. DOI

51. Mu C, Song Y, Huang W, et al. Flexible normal-tangential force sensor with opposite resistance responding for highly sensitive artificial skin. Adv Funct Mater 2018;28:1707503. DOI

52. Dagdeviren C, Su Y, Joe P, et al. Conformable amplified lead zirconate titanate sensors with enhanced piezoelectric response for cutaneous pressure monitoring. Nat Commun 2014;5:4496. DOI PubMed

53. Park DY, Joe DJ, Kim DH, et al. Self-powered real-time arterial pulse monitoring using ultrathin epidermal piezoelectric sensors. $A d v$ Mater 2017;29:1702308. DOI PubMed

54. Dagdeviren C, Yang BD, Su Y, et al. Conformal piezoelectric energy harvesting and storage from motions of the heart, lung, and diaphragm. Proc Natl Acad Sci U S A 2014;111:1927-32. DOI PubMed PMC

55. Dagdeviren C, Javid F, Joe P, et al. Flexible piezoelectric devices for gastrointestinal motility sensing. Nat Biomed Eng 2017;1:80717. DOI PubMed

56. Nguyen TD, Deshmukh N, Nagarah JM, et al. Piezoelectric nanoribbons for monitoring cellular deformations. Nat Nanotechnol 2012;7:587-93. DOI PubMed

57. Deng W, Yang T, Jin L, et al. Cowpea-structured PVDF/ZnO nanofibers based flexible self-powered piezoelectric bending motion sensor towards remote control of gestures. Nano Energy 2019;55:516-25. DOI

58. Yang Y, Pan H, Xie G, et al. Flexible piezoelectric pressure sensor based on polydopamine-modified BaTiO3/PVDF composite film for human motion monitoring. Sensors and Actuators A: Physical 2020;301:111789. DOI

59. Djohan N, Harsono B, Liman J, Hardhienata H, Husein I. The effect of indium oxide $\left(\mathrm{In}_{2} \mathrm{O}_{3}\right)$ dopant on the electrical properties of $\mathrm{LiTaO}_{3}$ thin film-based sensor. Ferroelectrics 2020;568:55-61. DOI

60. Wang M, Shi H, Ma T, et al. High-frequency vibration analysis of $\mathrm{LiTaO}_{3}$ piezoelectric plates excited by lateral electric fields produced by surface electrodes under viscous liquid loadings for sensing. Smart Mater Struct 2020;29:045004. DOI

61. Chen J, Liu H, Wang W, et al. High durable, biocompatible, and flexible piezoelectric pulse sensor using single-crystalline III-N thin film. Adv Funct Mater 2019;29:1903162. DOI

62. Kim N, Chang Y, Chen J, et al. Piezoelectric pressure sensor based on flexible gallium nitride thin film for harsh-environment and high-temperature applications. Sensors and Actuators A: Physical 2020;305:111940. DOI

63. Cheng S, Han S, Cao Z, Xu C, Fang X, Wang X. Wearable and ultrasensitive strain sensor based on high-quality GaN pn junction microwire arrays. Small 2020;16:e1907461. DOI PubMed

64. Kar E, Bose N, Dutta B, Mukherjee N, Mukherjee S. Ultraviolet- and microwave-protecting, self-cleaning e-skin for efficient energy 
harvesting and tactile mechanosensing. ACS Appl Mater Interfaces 2019;11:17501-12. DOI PubMed

65. Su Y, Chen C, Pan H, et al. Muscle fibers inspired high-performance piezoelectric textiles for wearable physiological monitoring. Adv Funct Mater 2021;31:2010962. DOI

66. Lu K, Huang W, Guo J, et al. Ultra-sensitive strain sensor based on flexible poly(vinylidene fluoride) piezoelectric film. Nanoscale Res Lett 2018;13:83. DOI PubMed PMC

67. Wang ZL, Wang AC. On the origin of contact-electrification. Materials Today 2019;30:34-51. DOI

68. Fan F, Tian Z, Lin Wang Z. Flexible triboelectric generator. Nano Energy 2012;1:328-34. DOI PubMed

69. Tian J, Chen X, Wang ZL. Environmental energy harvesting based on triboelectric nanogenerators. Nanotechnology 2020;31:242001. DOI PubMed

70. Wang X, Zhang Y, Zhang X, et al. A highly stretchable transparent self-Powered triboelectric tactile sensor with metallized nanofibers for wearable electronics. Adv Mater 2018;30:e1706738. DOI PubMed

71. Wang X, Zhang H, Dong L, et al. Self-powered high-resolution and pressure-sensitive triboelectric sensor matrix for real-time tactile mapping. Adv Mater 2016;28:2896-903. DOI PubMed

72. Sun H, Zhao Y, Wang C, et al. Ultra-stretchable, durable and conductive hydrogel with hybrid double network as high performance strain sensor and stretchable triboelectric nanogenerator. Nano Energy 2020;76:105035. DOI

73. Chun S, Son W, Kim H, Lim SK, Pang C, Choi C. Self-powered pressure- and vibration-sensitive tactile sensors for learning technique-based neural finger skin. Nano Lett 2019;19:3305-12. DOI PubMed

74. Mannsfeld SC, Tee BC, Stoltenberg RM, et al. Highly sensitive flexible pressure sensors with microstructured rubber dielectric layers. Nat Mater 2010;9:859-64. DOI PubMed

75. Zhang S, Wen L, Wang H, Zhu K, Zhang M. Vertical CNT-Ecoflex nanofins for highly linear broad-range-detection wearable strain sensors. J Mater Chem C 2018;6:5132-9. DOI

76. Lou Z, Chen S, Wang L, Jiang K, Shen G. An ultra-sensitive and rapid response speed graphene pressure sensors for electronic skin and health monitoring. Nano Energy 2016;23:7-14. DOI

77. Pan F, Chen S, Li Y, et al. 3D graphene films enable simultaneously high sensitivity and large stretchability for strain sensors. $A d v$ Funct Mater 2018;28:1803221. DOI

78. Boutry CM, Kaizawa Y, Schroeder BC, et al. A stretchable and biodegradable strain and pressure sensor for orthopaedic application. Nat Electron 2018;1:314-21. DOI

79. Chen S, Song Y, Ding D, Ling Z, Xu F. Flexible and anisotropic strain sensor based on carbonized crepe paper with aligned cellulose fibers. Adv Funct Mater 2018;28:1802547. DOI

80. Shi C, Zou Z, Lei Z, Zhu P, Zhang W, Xiao J. Heterogeneous integration of rigid, soft, and liquid materials for self-healable, recyclable, and reconfigurable wearable electronics. Sci Adv 2020;6:eabd0202. DOI PubMed PMC

81. Yu X, Xie Z, Yu Y, et al. Skin-integrated wireless haptic interfaces for virtual and augmented reality. Nature 2019;575:473-9. DOI PubMed

82. Xu F, Li X, Shi Y, et al. Recent developments for flexible pressure sensors: a review. Micromachines (Basel) 2018;9:580. DOI PubMed PMC

83. Wang C, Xia K, Wang H, Liang X, Yin Z, Zhang Y. Advanced carbon for flexible and wearable electronics. Adv Mater 2019;31:e1801072. DOI PubMed

84. Kang M, Kim J, Jang B, Chae Y, Kim JH, Ahn JH. Graphene-based three-dimensional capacitive touch sensor for wearable electronics. ACS Nano 2017;11:7950-7. DOI PubMed

85. Zeng X, Wang Z, Zhang H, et al. Tunable, ultrasensitive, and flexible pressure sensors based on wrinkled microstructures for electronic skins. ACS Appl Mater Interfaces 2019;11:21218-26. DOI PubMed

86. Gong S, Schwalb W, Wang Y, et al. A wearable and highly sensitive pressure sensor with ultrathin gold nanowires. Nat Commun 2014;5:3132. DOI PubMed

87. Liu Y, Zheng H, Zhao L, et al. Electronic skin from high-throughput fabrication of intrinsically stretchable lead zirconate titanate elastomer. Research (Wash D C) 2020;2020:1085417. DOI PubMed PMC

88. Qiu Y, Tian Y, Sun S, et al. Bioinspired, multifunctional dual-mode pressure sensors as electronic skin for decoding complex loading processes and human motions. Nano Energy 2020;78:105337. DOI

89. Hwang B, Zabeeb A, Trung TQ, et al. A transparent stretchable sensor for distinguishable detection of touch and pressure by capacitive and piezoresistive signal transduction. NPG Asia Mater 2019:11. DOI

90. Yang Y, Zhao G, Cheng X, Deng H, Fu Q. Stretchable and healable conductive elastomer based on PEDOT:PSS/natural rubber for self-powered temperature and strain sensing. ACS Appl Mater Interfaces 2021;13:14599-611. DOI PubMed

91. You I, Mackanic DG, Matsuhisa N, et al. Artificial multimodal receptors based on ion relaxation dynamics. Science 2020;370:961-5. DOI PubMed

92. Wang J, Jiang J, Zhang C, et al. Energy-efficient, fully flexible, high-performance tactile sensor based on piezotronic effect: Piezoelectric signal amplified with organic field-effect transistors. Nano Energy 2020;76:105050. DOI

93. Oh H, Yi GC, Yip M, Dayeh SA. Scalable tactile sensor arrays on flexible substrates with high spatiotemporal resolution enabling slip and grip for closed-loop robotics. Sci Adv 2020;6:eabd7795. DOI PubMed PMC

94. Guo $\mathrm{S}, \mathrm{Wu} \mathrm{K}, \mathrm{Li} \mathrm{C}$, et al. Integrated contact lens sensor system based on multifunctional ultrathin $\mathrm{MoS}_{2}$ transistors. Matter 2021;4:969-85. DOI PubMed PMC

95. Shi Q, He T, Lee C. More than energy harvesting - combining triboelectric nanogenerator and flexible electronics technology for enabling novel micro-/nano-systems. Nano Energy 2019;57:851-71. DOI 
96. Zhao J, Bu T, Zhang X, et al. Intrinsically stretchable organic-tribotronic-transistor for tactile sensing. Research (Wash D C) 2020;2020:1398903. DOI PubMed PMC

97. Yang GZ, Bellingham J, Dupont PE, et al. The grand challenges of Science Robotics. Sci Robot 2018;3:eaar7650. DOI PubMed

98. Kim J, Lee G, Heimgartner R, et al. Reducing the metabolic rate of walking and running with a versatile, portable exosuit. Science 2019;365:668-72. DOI PubMed

99. Zhang J, Fiers P, Witte KA, et al. Human-in-the-loop optimization of exoskeleton assistance during walking. Science 2017;356:12804. DOI PubMed

100. Valle G, Saliji A, Fogle E, Cimolato A, Petrini FM, Raspopovic S. Mechanisms of neuro-robotic prosthesis operation in leg amputees. Sci Adv 2021;7:eabd8354. DOI PubMed PMC

101. Li J, Esteban-Fernández de Ávila B, Gao W, Zhang L, Wang J. Micro/nanorobots for biomedicine: delivery, surgery, sensing, and detoxification. Sci Robot 2017;2:eaam6431. DOI PubMed PMC

102. Miskin MZ, Cortese AJ, Dorsey K, et al. Electronically integrated, mass-manufactured, microscopic robots. Nature 2020;584:557-61. DOI PubMed

103. Mishra S, Kim YS, Intarasirisawat J, et al. Soft, wireless periocular wearable electronics for real-time detection of eye vergence in a virtual reality toward mobile eye therapies. Sci Adv 2020;6:eaay1729. DOI PubMed PMC

104. Song K, Kim SH, Jin S, et al. Pneumatic actuator and flexible piezoelectric sensor for soft virtual reality glove system. Sci Rep 2019;9:8988. DOI PubMed PMC

105. Trung TQ, Lee NE. Flexible and stretchable physical sensor integrated platforms for wearable human-activity monitoringand personal healthcare. Adv Mater 2016;28:4338-72. DOI PubMed

106. Amjadi M, Kyung K, Park I, Sitti M. Stretchable, skin-mountable, and wearable strain sensors and their potential applications: a review. Adv Funct Mater 2016;26:1678-98. DOI

107. Ha M, Lim S, Ko H. Wearable and flexible sensors for user-interactive health-monitoring devices. J Mater Chem B 2018;6:4043-64. DOI PubMed

108. Zheng YL, Ding XR, Poon CC, et al. Unobtrusive sensing and wearable devices for health informatics. IEEE Trans Biomed Eng 2014;61:1538-54. DOI PubMed PMC

109. Shin J, Yan Y, Bai W, et al. Bioresorbable pressure sensors protected with thermally grown silicon dioxide for the monitoring of chronic diseases and healing processes. Nat Biomed Eng 2019;3:37-46. DOI PubMed

110. Wang Y, Zhu W, Deng Y, et al. Self-powered wearable pressure sensing system for continuous healthcare monitoring enabled by flexible thin-film thermoelectric generator. Nano Energy 2020;73:104773. DOI

111. Wang C, Li X, Hu H, et al. Monitoring of the central blood pressure waveform via a conformal ultrasonic device. Nat Biomed Eng 2018;2:687-95. DOI PubMed PMC

112. Liu Q, Huang J, Zhang J, et al. Thermal, waterproof, breathable, and antibacterial cloth with a nanoporous structure. ACS Appl Mater Interfaces 2018;10:2026-32. DOI PubMed

113. Zhang L, Jiang X, Jiang W, et al. Infrared skin-like active stretchable electronics based on organic-inorganic composite structures for promotion of cutaneous wound healing. Adv Mater Technol 2019;4:1900150. DOI

114. Ni X, Ouyang W, Jeong $\mathrm{H}$, et al. Automated, multiparametric monitoring of respiratory biomarkers and vital signs in clinical and home settings for COVID-19 patients. Proc Natl Acad Sci U S A 2021;118:e2026610118. DOI PubMed PMC

115. Araromi OA, Graule MA, Dorsey KL, et al. Ultra-sensitive and resilient compliant strain gauges for soft machines. Nature 2020;587:219-24. DOI PubMed 